\title{
Guest Editorial Advancements in Power Supply Current Testing
}

\author{
RAFIC Z. MAKKI
}

The University of North Carolina at Charlotte

As far back as the 1970's, the microelectronics test industry discovered a correlation between CMOS IC defects and the behavior of the power supply current. Companies utilized the premise that an IC exhibiting unusually high quiescent power supply current $\left(\mathrm{I}_{\mathrm{DDQ}}\right)$ levels is likely to be defective. They found that $\mathrm{I}_{\mathrm{DDQ}}$ can be especially useful in detecting defects in static CMOS circuits. The $I_{D D Q}$ in static CMOS circuits is a function of normal circuit leakages and is thus, normally, very low because the circuit has no direct path between PWR and Ground in the quiescent state. Defects which cause excessive leakage into the power supply bus can be detected by observing the resulting high $\mathrm{I}_{\mathrm{DDQ}}$ level. This simple technique grew in popularity because the power supply current is directly observable and can be used to detect defects that may not alter the logic behavior of the IC and could thus go undetected under logic testing. Such defects include gate-oxide shorts, double floating gate faults, defects which cause partially conducting (weak) transistors, and bridging faults. Tests are generated such that a path is established between PWR and Ground causing an elevated $\mathrm{I}_{\mathrm{DDQ}}$. For example, in the case of bridging faults, a path is established between PWR and GND via the bridged nodes.

Over the past decade, the research community has extensively studied $I_{D D Q}$ testing which resulted in a wealth of knowledge and numerous publications. The principal research issues include: finding a limiting value to $I_{D D Q}$ during testing (above which an IC is characterized as faulty), developing efficient built-in current (BIC) sensors to speed-up the test process, developing test pattern generation strategies and algorithms, developing techniques for designing $\mathrm{I}_{\mathrm{DDQ}}$ testable circuits vis-a-vis partitioning for BIC sensor insertion, efficiently integrating $\mathrm{I}_{\mathrm{DDQ}}$ with other test strategies such as logic testing, and data collection for reliability studies.

More recently, researchers have extended the idea of $I_{D D Q}$ to the transient portion of the power supply current. The principle is that both the quiescent and transient portions of the supply current carry important pieces of information that can be used to detect defects and improve IC quality.

This issue of the journal of VLSI Design presents both new approaches to power supply current testing and additional data on $\mathrm{I}_{\mathrm{DDQ}}$ testing. The following notation is used throughout this special issue

$\mathrm{i}_{\mathrm{DD}}$ : denotes the complete supply current

$\mathrm{I}_{\mathrm{DDQ}}$ : denotes the peak value of the quiescent supply current

$\mathrm{I}_{\mathrm{DDT}}$ : denotes the peak value of the transient supply current 
parity and two-rail checkers such that they receive all code words irrespective of whether the functional part of the self-checking system produces all code words or not. An internal LFSR (Linear Feedback Shift Register) is used to provide all the code words to a checker.

Until very recently most of the work on selfchecking design has focused on gate-level circuits, very little have been reported on self-checking design at the transistor-level. Since gate-level circuits are implemented at the transistor-level, research in the design of circuits that are selfchecking for exclusively transistor-level faults e.g. transistor stuck-on, transistor stuck-off, bridging, breaks has become extremely important. Authors Yeong-Ruey Shieh and Cheng-Wen Wu deal with a design technique for CMOS circuits such that these circuits are partially strongly code-disjoint (PSCD) for transistor stuck-on faults as well as for bridging faults. They have illustrated their technique by designing a PSCD 4-out-of-8 code checker circuit.

As mentioned previously a major objective of self-checking design is to enhance the reliability of a system. Jien-Chung Lo analyzes the reliability of self-checking circuits. His paper shows that the reliability of a system which uses duplicate- and -compare approach to achieve self-checking, is significantly higher than that can achieved by a TMR (Triple Modular Redundancy) scheme. An important conclusion of the paper is that depending on the application a self-checking circuit can be either self-checking or self-testing, but not necessarily be both.

The final paper, written by F. Vainstein, focuses on a technique for on-line checking of numerical functions. This technique is especially useful for functions with large number of arguments.

\section{Acknowledgment}

I would like to thank the following individuals for their careful and timely reviews of the submitted papers: A. Al-Khalili, G. Blakely, M. Blum, F. Busaba, M. Goessel, N. Kanapoulous, M. Karpovsky, J. S. Lo, M. Nicolaidis, S. Tarnick, F. Vainstein, A. Walker, C. W. Wu, and Y. Zhou.

\section{Author's Biography}

Dr. Parag K. Lala is a Research Professor in the Department of Electrical Engineering, North Carolina A\&T State University. He received an M.Sc (Eng.) degree from King's College, London, and a Ph.D degree from the City University of London. $\mathrm{He}$ is the author/co-author of more than eighty papers. He is also the author of three books: Fault-Tolerant \& Fault-Testable Hardware Design (Prentice-Hall, 1985), Digital System Design using PLDs (Prentice-Hall, 1990), and Practical Digital Logic Design and Testing (Prentice-Hall, 1996). Dr. Lala has recently been awarded the outstanding educator award by the IEEE North Carolina Central section for his contributions to fault tolerant computing. He served in the program committee of the 1st IEEE International On-Line Testing Workshop held in Nice, France in July, 1995. He is a Fellow of the IEE, and a Senior Member of the IEEE. 

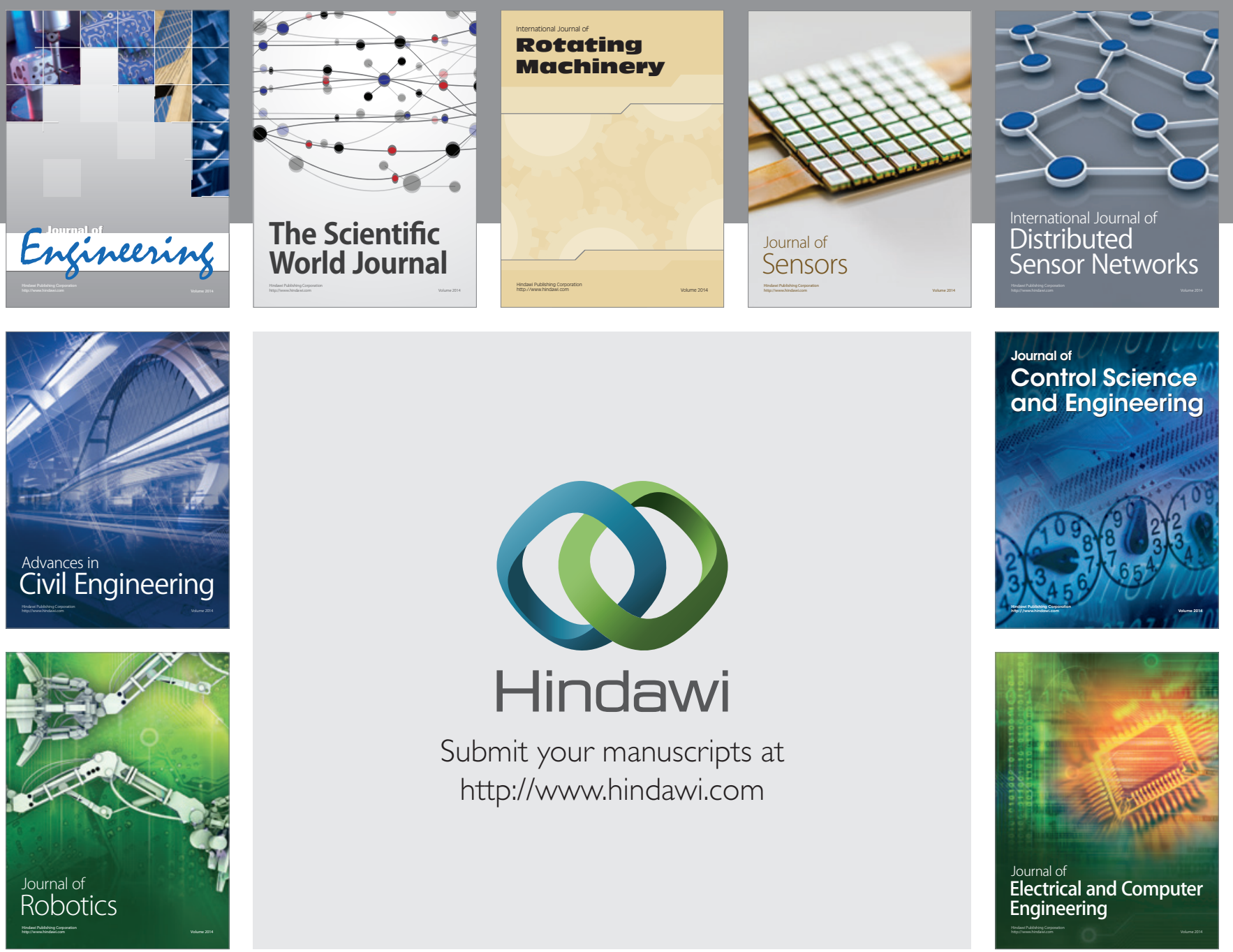

Submit your manuscripts at

http://www.hindawi.com
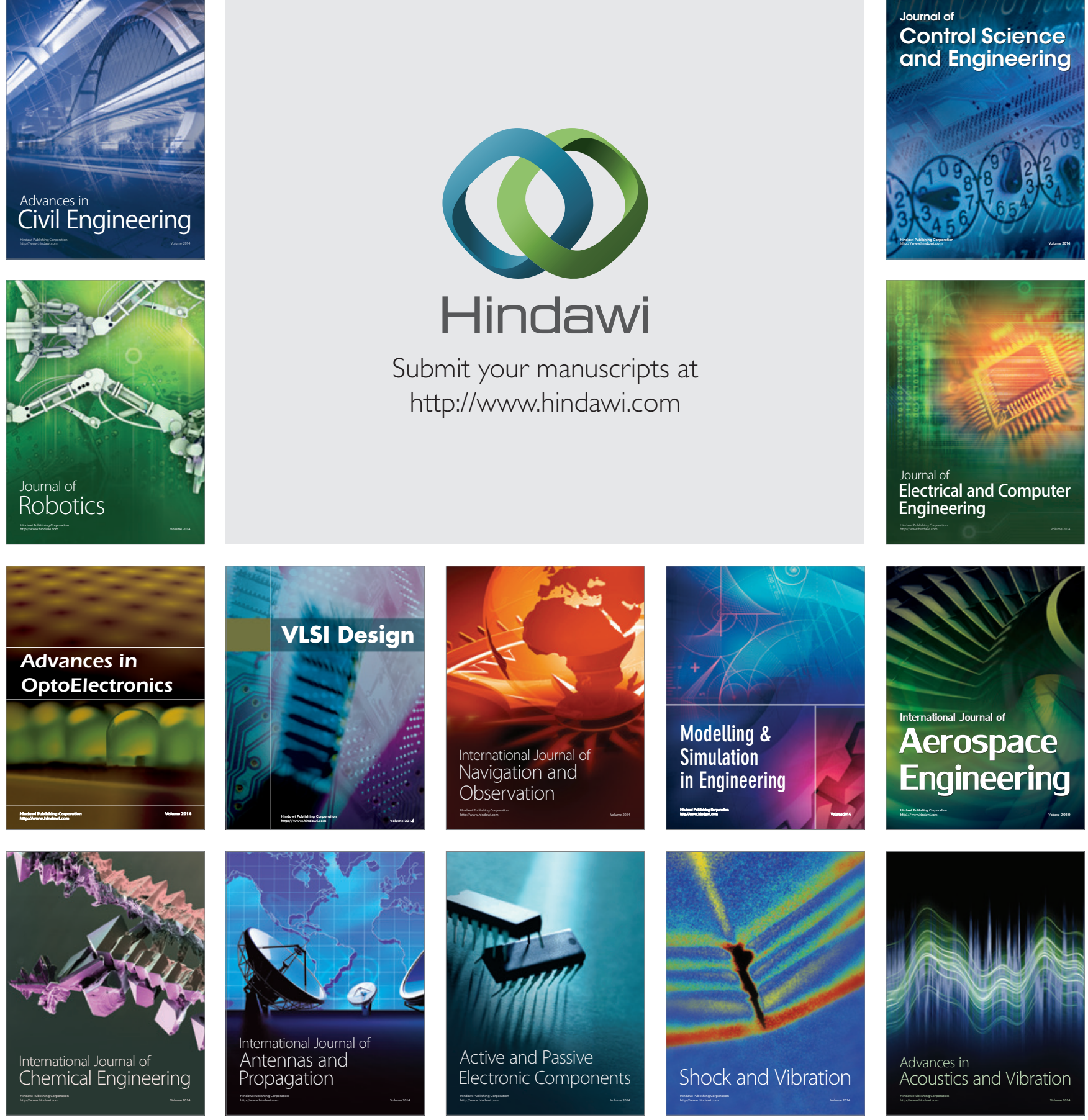